\title{
The Effect of Mirror Neurons Stimulation on Syntax Development of Female Persian Autistic Children
}

\author{
Hanieh Yarmand ${ }^{1}$, Hassan Ashayeri ${ }^{2}$, Arsalan Golfam ${ }^{3} \&$ Hayat Ameri $^{3}$ \\ ${ }^{1}$ Faculty of Foreign Languages, Islamic Azad University, Science and Research Branch, Tehran, Iran \\ ${ }^{2}$ Faculty of Rehabilitation Sciences, Iran University of Medical Sciences, Tehran, Iran \\ ${ }^{3}$ Faculty of Humanities, Tarbiat Modares University, Tehran, Iran \\ Correspondence: Hanieh Yarmand, Faculty of Foreign Languages, Islamic Azad University, Science and \\ Research Branch, Tehran, Iran. E-mail: h.yarmand@srbiau.ac.ir
}

Received: June 28, 2016 Accepted: August 2, 2016 Online Published: November 23, 2016

doi:10.5539/ijel.v6n6p67 URL: http://dx.doi.org/10.5539/ijel.v6n6p67

\begin{abstract}
One common language disorder in autistic children is syntax disorder. The current research aims to examine the relationship between mirror neurons stimulation by intentional movements imitation and verbal imitation with syntax skill development in autistic children. This research was performed using an experimental applied design and convenience sampling method. First, the researcher designed a functional and easy model for autistic children rehabilitation based on intentional movement imitation and verbal imitation; Then, using TPR (Total Physical Response) method. A pilot study was conducted on a Persian-speaking autistic girl aged 7 in 12 sessions for four weeks in Iran University of Medical Sciences, School of Rehabilitation Sciences, and effective positive effects were observed. Then, 8 Persian-speaking autistic children were examined in terms of entrance criteria, and finally, 5 autistic girls aged 5-8 were selected and underwent training courses for 42 sessions over 14 weeks (3 20-30 minute sessions). In order to examine syntax skills of the subjects including grammatical understanding, sentence imitation and grammatical completion, before and after intervention, TOLD-P3 test was used. Each subject, as his/her Control was examined before and after intervention. Furthermore, two months after a 14-week stopping period, all the subjects were re-examined using TOLD-P3 test, and finally, results stability was examined. The research findings were analyzed using ANOVA test. The findings show that mirror neurons stimulation in autistic children through intentional movement imitation and verbal imitation has a positive effect on syntax skill improvement on these children thereby facilitating their verbal communication.
\end{abstract}

Keywords: autism, mirror neurons system, intentional movements imitation, verbal imitation, language improvement

\section{Introduction}

Recent developments in neurological research have given rise to discovery of new neurons in monkey's brain called mirror neurons (Rizzolatti \& Arbib, 1998); (Rizzolatti et al., 2009); (Buccino \& Solodkin, 2012) Ramachandran (2000), a leading Indian Neurologists in America, considers the newly-discovered mirror neurons the greatest discovery of human history after discovery of "DNA". He assumes that "mirror neurons will do for psychology what DNA did for biology: they will provide a unifying framework and help explain a host of mental abilities that have hitherto remained mysterious and inaccessible to experiments" (Wilson \& Knoblich, 2005). Mirror neurons were first discovered in the early 1990s, when a team of researchers in Rizzolatti lab in Parma University were working on macaque monkeys. These motor neurons have special properties in area F5 of macaque monkey's brain. These neurons fired both when the monkey executed a movement, such as grasping a banana, and also when the monkey watched the experimenter execute that very same movement (when the experimenter grasps that banana) (Arbib, 2012). Rizzolatti et al. called these neurons as mirror neurons since executing that action and observing that action have the same reflection in the brain (Figure 1). 


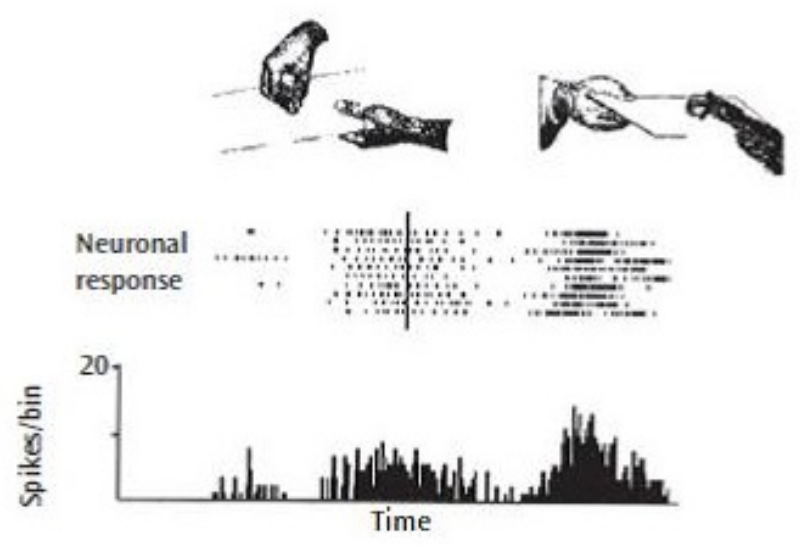

Figure 1. An example of mirror neurons

The upper part of the picture shows the movement relating to grasping the banana. The lower part shows the neurons response.

Further examinations showed that mirror neurons exist in some birds and human besides macaque monkeys. In 1995, the first evidence for the existence of mirror neurons in humans was provided by Fadiga, Fogassi, Pavesi and Rizzolatti. Results of different researches show that the location of these neurons in human's brain overlaps with that of monkey (Aziz-Zadeh et al., 2006). Evidence shows that the motor sector of Broca's area in human's brain is located at inferior frontal cortex. This area is called Brodmann's area 44. According to the recent studies, there seems to be a homology between Brodmann area 44 in humans and the monkey area F5. Electrophysiological evidence suggests the presence of mirror mechanism in these areas. Studies of intracranial magnetic stimulation (TMS) (Fadiga et al., 1995) (Gangitano et al., 2001), of Electroencephalography (EEG) (Cochin et al., 1999) and of Magnetoencephalography (MEG) (Hari et al., 1998) suggest that human motor system in Broca's area has mirror features. In other words, the investigations show that mirror neurons system in humans is located in Broca's area (Arbib, 2012). Broca's area is located at inferior frontal cortex of human brain (Hickok et al., 2011). This area is responsible for motor planning of speech and language. A damage to this area impairs the motor and language part of the spoken language (Stemmer \& Whitaker, 2008). Following the discovery of mirror neurons at Broca's area of human brain, some issues were raised regarding different tasks of human brain including movement understanding, imitation, word understanding, language learning, as well as the evolution of language and their association with mirror neurons. Mirror neurons system is known to play an important role in understanding the actions of others and is necessary for human ability to learn through observation and imitation (Van Gog et al., 2009). Many researchers have shown that mirror neurons system is seriously impaired in autistic individuals; as a result, autistic individuals have trouble imitating others. As we know, imitation in human plays an important role in understanding actions and activities, especially in the process of learning language; therefore, preparation and execution of intervention program which focus on imitative skills of these patients should be taken into account. Studies suggest that the reported number of autism has increased in individuals (Tager-Flusberg et al., 2009). Currently, the prevalence of autism has been reported to be 6 per 1000 people (Toth et al., 2007), Although the rate of autistic people has also increased in Iran and despite numerous reports about autistic children's behavioral disorders, there are not enough clinical and intervention strategies to improve their verbal skill. Therefore, the current paper aims to examine the effect of mirror neurons system stimulation through intentional movement imitation and verbal imitation on syntax skill development of autistic children.

This research is an interdisciplinary research including such areas as cognitive sciences, clinical linguistics, psycholinguistics, neurolinguistics, performed in order to solve language disorders.

\section{Methodology}

The intervention and data collection stages of this study were performed in Iran University of Medical Sciences, School of Rehabilitation Sciences for 14 weeks. This study is of empirical, practical, non-invasive and researcher-made type using a pretest-posttest design. The samples consist of 5 autistic girl aged 5-8 selected from the samples available in special schools and clinics for autistics children in Tehran. Since not many autistic 
children are girls and this disease occurs more frequently in boys and due to highly limited access to these children, convenience sampling method was used. Accordingly, 5 autistic girls that matched inclusion criteria were selected for intervention. Selection criteria of the children to participate in this study were as follows:

1) they should be girl;

2) they should be diagnosed with autism by psychiatrists;

3) they should be 5-8 years old;

4) they parents should be interested in letting their children participate in the study;

5) they should not use any medication;

6) they should not have any organic disease;

7) they should not simultaneously undergo any other medication or intervention;

8) they should have the ability to imitate at least one movement; such as sitting.

The existence of each of the following factors would lead to the exclusion of the subject:

1) either the child or the parents are not interested in participating the study;

2) child's absence for more than three sessions.

In order to perform this study, the researchers designed an easy and functional model for rehabilitation of autistic children based on intentional movement imitation and verbal imitation. "Early Language Development" written by Mawhinney and Mc Teague was used to design this model. The words were divided into three groups in order to prepare suitable sentences: object words, action words, and concept words. Object words such as "ball, scarf" refer to a specific object; action words refer to a specific action such as "sitting, standing", and concept words such as "under, above" include abstract concepts (Mawhinney \& Mc Teague, 2004). Sentences are designed such that they include imitative movements associated with hand, foot, and head, imitation of performing an act on objects as well as oral-facial imitation.

In order to comply with ethical considerations, the subjects' parents were asked to participate in a session to ensure them that the information of the children and their parents remains confidential; moreover, they were given some information about the course to be assured that the research will impose no risk on their children. Then, a written consent was signed by the parents. Furthermore, a questionnaire asking for personal information of the children was completed by the parents. The subjects; i.e., 5 monolingual Persian-speaking autistic girls aged 5-8, underwent 42 20-30 minute sessions per week over 14 weeks. The subjects were asked to perform two imitation assignments in each session. The examiner trained the children using TPR based on which the child can learn the movements by both observing and performing them. Over the entire course, the child was asked to imitate 91 different movements from the examiner; moreover, the child was also supposed to repeat the sentences associated with these movements uttered by the examiner. In order to help the child understand the assignment, at the beginning of each session, two simple imitative assignments were conducted by the examiner for a few times, and then the child was asked to repeat them. In case of not imitating the actions, the child was encouraged by the examiner to imitate the actions by moving the child's body to represent the action. After making sure that the children understood the process of imitation, the examiner presented the imitative actions of that session. However, it should be mentioned that the imitative assignments of the previous session were reviewed at the beginning of each session and in case of performing them correctly; new assignments were provided by the examiner. The examiner performed the action three times for the child and asked the child to repeat the act by uttering the imperative sentence "...do what I do" (TPR method). In case of responding to an assignment, the child received the next item; otherwise, the examiner repeated that action. It was done at most for three times. The child was encouraged by receiving verbal awards or she was given some snacks. To perform imitative assignments, the researchers are inspired by Rogers et al. (2003). In this study, the required data was collected by performing an interview with the family, completing the questionnaire, registering the data of each session in special forms, performing tests, recording the children's response before and after intervention, as well as videotaping training sessions.

\section{Data Collection Tools}

The tools used for collecting data include:

\subsection{Questionnaire}

Primary information about the children including: personal information such as age, sex, date of birth, dominant hand, history of seizures, medications, medical history, family history, as well as the extent of language ability 
were collected using a questionnaire designed based on Manolson model.

\subsection{Test of Language Development TOLD-P: 3}

TOLD-P: 3 test is one of the most common and comprehensive tests in the field of measuring children language growth. This test was firstly published in 1977 by the name of TOLD having five sub-scales. In 1982, following important changes, this test was changed to "TOLD-P" (Test of Language Development- Primary). The reason of including the letter $p$ which stands for "primary" was to distinguish it from its counterpart called "Told-I" (Test of Language Development- Intermediate) which was prepared for children aged 8-12. In 1988, the second edition of "TOLD-P" called "TOLD-P: 2" was published to which great and important changes were applied to improve the test. In 1997, the test designers applied some changes to the test based on their experiences and suggestions by those individuals who had previously used "TOLD-P: 2", thereby "TOLD-P: 3" was formed for children aged 4-8. This test is based on a two-dimensional model including: 1) components of linguistic system such as listening, organizing and speaking, and 2) components of linguistic features such as semantics and syntax. The two-dimensional model of the interest includes 9 subscales; 6 of which are related to semantics and syntax which are among the main subscales and 3 of which are related to phonemics and are additional subscales. The semantic components are measured using 3 subscales including picture vocabularies, relative vocabularies and oral vocabularies.

The syntax component is measured using 3 subscales including grammatical understanding, sentence imitation and grammatical completion. Finally, the phonetic component is measured using 3 subscales including word distinguishing, phonological analysis and word generation. Table 1 illustrates a two-dimensional model used in building TOLD-P: 3.

Table 1. A two-dimensional model used in building TOLD-P: 3

\begin{tabular}{llll}
\hline Linguistic Systems & & & \\
\hline Linguistic Components & Listening & Organizing & Speaking \\
Semantics & Picture vocabulary & Relative vocabulary & Oral vocabulary \\
Syntax & Grammatical understanding & Sentence imitation & Grammatical completion \\
Phonetics & Word distinguishing & Phonetic analysis & Word generation \\
\hline
\end{tabular}

In this research, to investigate the syntax skill of these children, before and after intervention, three subscales were used including: grammatical understanding, sentence imitation and grammatical completion. As can be seen in Table 1, syntax part of test of language development consists of three subscales including grammatical understanding, sentence imitation and grammatical completion which evaluate the subject's grammar in form of items. This test was completed at three stages in the presence of parents as well as neuroscientist and linguist by observing and interviewing. Each of the subscales took about 15-20 minutes. Grading method was as follows: grade 1 for each correct answer and 0 for each false answer. The raw score of the subject was obtained from the total scores obtained in each subscale. Notably, the maximum permissible error in this test is fixed; more precisely, if the subject loses the score of 5 consecutive items in each of the subscales, execution of that subscale will be stopped.

\subsection{Camera and Camcorder}

Camera and camcorder were required to prepare images and record the medical training session of the children. A mobile camera of Samsung SIII 8MP was used rather than a camera. In order to film the sessions, Canon camera was used. However, it should be mentioned that it was impossible to record all sessions due to children's sensitivity.

\subsection{Toys and Entertainment}

A number of finger puppets, toys, comic books, and balls as well as some play dough were prepared to entertain the children. Moreover, the room was decorated with happy colorful posters.

\subsection{Flash Cards}

Flash cards with color images were used to teach object words, if necessary.

\section{Results}

This research was conducted on 5 autistic children with an average age of 7 years and 8 months. Table 2 lists mean, standard deviation (SD), maximum and minimum pretest-posttest scores of the subscales including 
subjects' grammatical understanding, sentence imitation, grammatical completion, and syntax.

Table 2. A comparison of mean, SD, maximum and minimum pre-test-post-test scores of the subscales including subjects' grammatical understanding, sentence imitation, grammatical completion, and syntax

\begin{tabular}{|c|c|c|c|c|c|c|c|c|c|c|c|c|c|c|}
\hline \multirow[t]{2}{*}{ variables } & \multirow[t]{2}{*}{$\mathrm{N}$} & \multicolumn{4}{|c|}{ Pre-test } & \multicolumn{2}{|c|}{ Confidence } & \multicolumn{4}{|c|}{ Post-Test } & \multicolumn{2}{|c|}{ Confidence } & \multirow[t]{2}{*}{ sig } \\
\hline & & mean & $\mathrm{sd}$ & $\min$ & $\max$ & up & low & mean & $\mathrm{sd}$ & $\min$ & $\max$ & up & low & \\
\hline $\begin{array}{l}\text { Grammatical } \\
\text { understanding }\end{array}$ & 5 & 2.2 & 1.09 & 1 & 4 & 3.56 & 0.83 & 5.6 & 1.14 & 4 & 7 & 7.01 & 4.18 & 0.001 \\
\hline $\begin{array}{l}\text { Sentence } \\
\text { imitation }\end{array}$ & 5 & 3.6 & 0.89 & 3 & 5 & 4.71 & 2.48 & 6 & 1.58 & 4 & 8 & 7.96 & 4.03 & 0.018 \\
\hline $\begin{array}{l}\text { Grammatical } \\
\text { completion }\end{array}$ & 5 & 2.2 & 0.83 & 1 & 3 & 3.23 & 1.16 & 4.6 & 1.14 & 3 & 6 & 6.01 & 3.18 & 0.005 \\
\hline Syntax & 5 & 8 & 1.22 & 6 & 9 & 9.52 & 6.47 & 16.2 & 3.03 & 12 & 20 & 19.9 & 12.43 & 0.001 \\
\hline
\end{tabular}

As can be seen in the above Table, the obtained results suggest that there is a significance difference between subjects' pretest-posttest scores of grammatical understanding $(\mathrm{p}<0.001)$, sentence imitation $(\mathrm{p}<0.018)$, grammatical completion $(\mathrm{p}<0.005)$ and finally syntax $(\mathrm{p}<0.001)$. Therefore, the subjects were found to show a significant improvement in development of grammatical understanding, sentence imitation, grammatical completion and syntax. In other words, the intervention method based on training verbal imitation and intentional movement imitation has been found to be effective in improving subjects' syntax skill. Graphs 1, 2, 3, and 4 show the pretest-posttest results obtained from subjects' development of grammatical understanding, sentence imitation, grammatical completion and syntax.

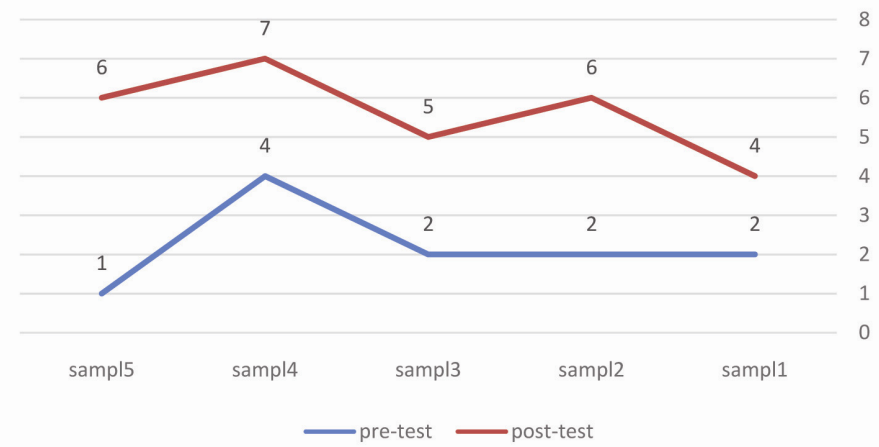

Graph 1. Pretest-posttest results of grammatical understanding skill of all subjects

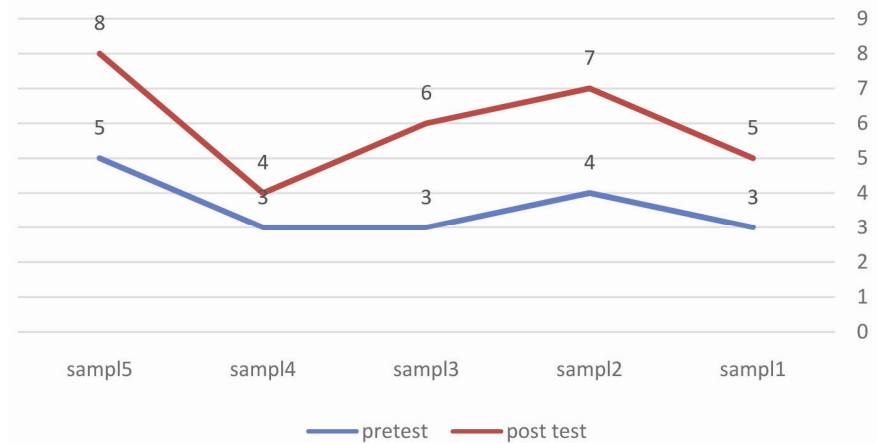

Graph 2. Pretest-posttest results of sentence imitation skill of all subjects 


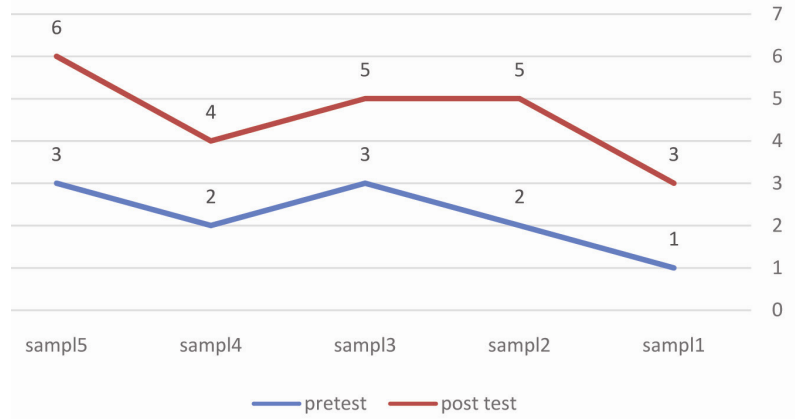

Graph 3. Pretest-posttest results of grammatical completion skill of all subjects

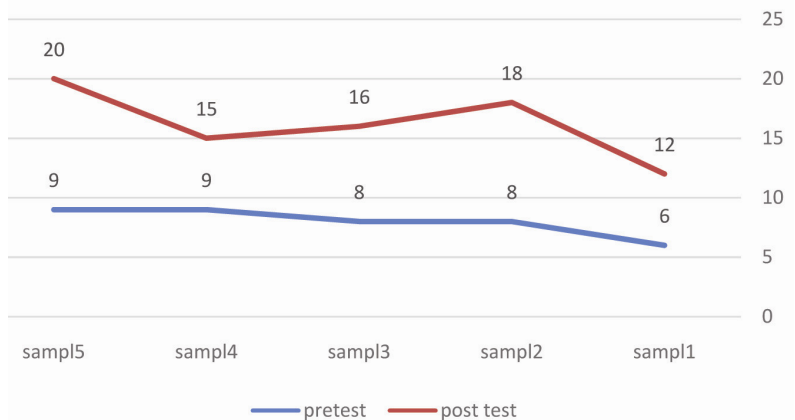

Graph 4. Pre-test \& post-test results of syntax skill of all subjects

\section{Discussion}

As evidence suggests, in autism, whereby the individual has significant communication impairment from childhood being unable to understand desires, emotions, needs and verbal concepts of others and has difficulty with social interaction, mirror neurons system is strongly impaired. In 1999, Ritta Haris' group presented the hypothesis of a deficient MNS in autistic individuals for the first time (Avikainen et al., 1999). Two years after this hypothesis was raised, Williams et al. published the first article on imitation, mirror neurons and autism (Williams et al., 2001). They highlighted the inability of the autistic individuals to imitate in the early years of life. Following publication of this article, numerous studies were conducted to verify their claim (Hadjikhani, 2007). One of the tools to ensure the health of mirror neurons systems in humans is EEG and its fluctuations i.e. MU fluctuations. In a study, a comparison was made between 14 autistic children and 14 healthy children, who are similar in terms of age and sex. The findings suggested that gray area of the brain in mirror neurons area in autistic children is significantly less than that of the healthy children. The degree of thinning in cortical areas in mirror neurons was correlated with autism symptom severity (Oberman et al., 2005). Lepage \& Theorete et al. performed a study on autistic individuals using EEG obtaining similar results (Lepage \& Théoret, 2006). In 2005, Theorete et al. examined primary motor cortex of autistic individuals using TMS; their findings also confirmed the theory of mirror neurons system impairment in autistic individuals (Théoret et al., 2005). The findings of two researches performed using FMRI also confirmed the above theory (Hadjikhani et al., 2007).

In 2009, Rizzolatti, Fogassi, \& Gallese, in their joint paper published in the book entitled "The Cognitive Neurosciences" showed that some deficiencies in autistic individuals arise from mirror neurons system impairment ( Rizzolatti et al., 2009). Therefore, it is supposed that stability and health of this nervous system provide the foundation for development of social skills such as language learning; therefore, a treatment which focuses on teaching imitation skills can be an effective method for improving socio-cognitive aspects of autistic individuals (Hadjikhani, 2007). Wallen \& Bulkeley, for example, showed that three sessions of adult imitation increased some appropriate social behaviors of young children with Autism (2006). Ingersol \& Lalonde, examined 4 autistic children. They investigated the impact of object and gesture imitation training on language use in children with autism disorder. They used Reciprocal Imitation Training (RIT) for object and gesture imitation training. Findings showed that application of Reciprocal Imitation Training (RIT) method in object and gesture imitation training improves language of autistic children (2010). Steinmeyer, trained 4 autistic children for 6 weeks. Over this period, he taught gestural, oral (e.g. pressing lips or smiling) and verbal imitation to the 
children. The children underwent a training session per week, one 30-minute session by the parents and one 30 -minute session by the therapist. The findings confirmed the importance of imitation therapy as an effective intervention method in improvement of social skills of these patients (Steinmeyer, 2013). Recent studies also confirm this hypothesis. The results of neurological studies show that neuroplasticity can not only be in form of the healthy hemisphere function rather than the damaged one, it can also be in form of reorganization of the representation of motion in the affected hemisphere. Action observation and imitation leads to organizational changes in mirror neurons system of the brain (Fadiga et al., 1995). These changes are the underlying factor for learning motion skills such as language (Fadiga et al., 1995). In general, it can be said that training intentional movement imitation has a positive effect on both visual agent through observing the action and individual's motion planning ability. Mirror neurons are activated by observation of actions; they are also activated during performing motion actions. In other words, it can be said that observation of actions and action imitation stimulate mirror neurons system. Mirror neurons system stimulation in Broca's area reactivates this area in the left hemisphere thereby developing speech and language skills (Small, 2009). Studies conducted by brain imaging show that Broca's area located at inferior frontal cortex as well as Wernicke's region located at superior temporal cortex are the key areas for processing syntax in the brain. The results of these studies highlight the role of these areas in processing syntax at different levels. Broca's area which involves mirror neurons system is responsible for speech and language motion planning while being active during generating and understanding complex syntactic structures; moreover, it is a necessary agent in generating and understanding these structures (Pulvermüller \& Fadiga, 2010). As a result, mirror neurons system stimulation at Broca's area can have a positive effect on development of syntactic skills. The findings of the current research show that autistic children showed significant improvement in syntactic skill. According to neuroplasticity and the critical role that Broca's area plays in generating and understanding complex syntactic structures, a change in syntactic structure of the children seemed likely.

\section{Conclusion}

A common linguistic disorder in autistic children is syntactic language impairment. The current research findings show that intentional movements imitation and verbal imitation, which were presented as a practice during the intervention course, give rise to development of syntactic skill of the autistic children. In general, the findings of this research are consistent with the results of other studies such as those performed by Ingersoll \& Lalonde (2010) and Steinmeyer (2013), which all show the effect of mirror neurons system stimulation on language development of autistic children. Carvalho et al. studied the role of mirror neurons system in rehabilitation of stroke patients. They found that mirror therapy by activating mirror neurons system through imitation improves motion activities of these patients (Carvalho et al., 2013). Regardless of differences in terms of disorders of the current research and the one performed by Carvalho et al. the effect of mirror neurons system stimulation on improvement of verbal ability can be observed.

The findings of the current research show that the average of syntax skill growth in all subjects has increased from 8 (pretest) to 16 (posttest) which caused a significant change in all the subjects $(\mathrm{p}<0.001)$. Furthermore, syntax skill growth level improved in each individual subject. In general, mirror neurons system stimulation created a significant improvement in syntactic skill level of autistic children, and specifically improved the grammatical sub-skills of this group including grammatical understanding $(p<0.001)$, sentence imitation $(\mathrm{p}<0.018)$ and grammatical completion $(\mathrm{p}<0.005)$. Therefore, the effect of mirror neurons system stimulation on rehabilitation of autistic children's verbal condition, in comparison with the results of this research and other researches even with different variables and methods, was evaluated to be positive. Given that all the subjects, before the course started, had undergone traditional speech therapy (at least 6 months) with no significant changes in their speech, it can be claimed that intentional movements imitation and verbal imitation improved the speech of all subjects. Moreover, given that the intervention course in this research took 14 weeks, it can be said that developmental changes as a result of increase in abilities over the course of time, had no considerable effect on research results. According to the research findings, application of this method has been found to be effective; therefore, it is recommended that, in case of similar research condition, the effect of mirror neurons system stimulation on effectiveness of theory of mind in autistic children be examined.

The main limitation of this study was the limited number of the subjects. Since not many autistic children are girls and this disease occurs more frequently in boys and due to highly limited access to these children, it was quite difficult to find subjects who matched the inclusion criteria for the study. Due to the limited number of the subjects, it was impossible to select a Control group, therefore, each subject was considered to be her own Control. 


\section{Acknowledgments}

We would like to highly appreciate the kind cooperation of autistic children's families.

\section{References}

Arbib, M. A. (2012). How the brain got language: The mirror system hypothesis (Vol. 16). Oxford: Oxford University Press. http://dx.doi.org/10.1093/acprof:osobl/9780199896684.001.0001

Avikainen, S., Kulomäki, T., \& Hari, R. (1999). Normal movement reading in Asperger subjects. Neuroreport, 10(17), 3467-3470. http://dx.doi.org/10.1097/00001756-199911260-00001

Aziz-Zadeh, L., Koski, L., Zaidel, E., Mazziotta, J., \& Iacoboni, M. (2006). Lateralization of the human mirror neuron system. The Journal of Neuroscience, 26(11), 2964-2970. http://dx.doi.org/10.1523/JNEUROSCI.2921-05.2006

Cochin, S., Barthelemy, C., Roux, S., \& Martineau, J. (1999). Observation and execution of movement: similarities demonstrated by quantified electroencephalography. European Journal of Neuroscience, 11(5), 1839-1842. http://dx.doi.org/10.1046/j.1460-9568.1999.00598.x

Fadiga, L., Fogassi, L., Pavesi, G., \& Rizzolatti, G. (1995). Motor facilitation during action observation: a magnetic stimulation study. Journal of Neurophysiology, 73(6), 2608-2611.

Gangitano, M., Mottaghy, F. M., \& Pascual-Leone, A. (2001). Phase-specific modulation of cortical motor $\begin{array}{lllll}\text { output during movement } & \text { observation. Neuroreport, 12(7), }\end{array}$ http://dx.doi.org/10.1097/00001756-200105250-00038

Hadjikhani, N. (2007). Mirror neuron system and autism. In Progress in Autism Research (pp. 151-166). Nova Science Publishing Inc.

Hadjikhani, N., Joseph, R. M., Snyder, J., \& Tager, F. H. (2007). Abnormal activation of the social brain during face perception in autism. Human brain mapping, 28(5), 441-449. http://dx.doi.org/10.1002/hbm.20283

Hari, R., Forss, N., Avikainen, S., Kirveskari, E., Salenius, S., \& Rizzolatti, G. (1998). Activation of human primary motor cortex during action observation: a neuromagnetic study. Proceedings of the National Academy of Sciences, 95(25), 15061-15065. http://dx.doi.org/10.1073/pnas.95.25.15061

Hickok, G., Costanzo, M., Capasso, R., \& Miceli, G. (2011). The role of Broca's area in speech perception: evidence from aphasia revisited. Brain and Language, 119(3), 214-220. http://dx.doi.org/10.1016/j.bandl.2011.08.001

Ingersoll, B., \& Lalonde, K. (2010). The impact of object and gesture imitation training on language use in children with autism spectrum disorder. Journal of Speech, Language, and Hearing Research, 53(4), 1040-1051. http://dx.doi.org/10.1044/1092-4388(2009/09-0043)

Lepage, J. F., \& Théoret, H. (2006). EEG evidence for the presence of an action observation-execution matching system in children. European Journal of Neuroscience, 23(9), 2505-2510. http://dx.doi.org/10.1111/j.1460-9568.2006.04769.x

Oberman, L. M., Hubbard, E. M., McCleery, J. P., Altschuler, E. L., Ramachandran, V. S., \& Pineda, J. A. (2005). EEG evidence for mirror neuron dysfunction in autism spectrum disorders. Cognitive Brain Research, 24(2), 190-198. http://dx.doi.org/10.1016/j.cogbrainres.2005.01.014

Pulvermüller, F., \& Fadiga, L. (2010). Active perception: sensorimotor circuits as a cortical basis for language. Nature Reviews Neuroscience, 11(5), 351-360. http://dx.doi.org/10.1038/nrn2811

Rizzolatti, G., \& Arbib, M. A. (1998). Language within our grasp. Trends in Neurosciences, 21(5), 188-194. http://dx.doi.org/10.1016/S0166-2236(98)01260-0

Rizzolatti, G., Fogassi, L., \& Gallese, V. (2009). The mirror neuron system: a motor-based mechanism for action and intention understanding. The Cognitive Neuroscience, IV, 625-640. http://dx.doi.org/10.1002/9780470478509.neubb001017

Rogers, S. J., Hepburn, S. L., Stackhouse, T., \& Wehner, E. (2003). Imitation performance in toddlers with autism and those with other developmental disorders. Journal of Child Psychology and Psychiatry, 44(5), 763-781. http://dx.doi.org/10.1111/1469-7610.00162

Small, S. (2009). A Biological basis for Aphasia Treatment: Mirror Neurons and Observation-Execution Matching. Poznań Studies in Contemporary Linguistics, 45(2), 313-326. http://dx.doi.org/10.2478/v10010-009-0017-3 
Small, S. L., Buccino, G., \& Solodkin, A. (2012). The mirror neuron system and treatment of stroke. Developmental Psychobiology, 54(3), 293-310. http://dx.doi.org/10.1002/dev.20504

Steinmeyer, N. (2013). Imitation Therapy in Young Children with Autism. Graduate Annual, 1(1), 11.

Stemmer, B., \& Whitaker, H. A. (2008). Handbook of the Neuroscience of Language. New York: Academic Press.

Tager-Flusberg, H., Rogers, S., Cooper, J., Landa, R., Lord, C., Paul, R., ... Yoder, P. (2009). Defining spoken language benchmarks and selecting measures of expressive language development for young children with autism spectrum disorders. Journal of Speech, Language, and Hearing Research, 52(3), 643-652. http://dx.doi.org/10.1044/1092-4388(2009/08-0136)

Théoret, H., Halligan, E., Kobayashi, M., Fregni, F., Tager-Flusberg, H., \& Pascual-Leone, A. (2005). Impaired motor facilitation during action observation in individuals with autism spectrum disorder. Current Biology, 15(3), R84-R85. http://dx.doi.org/10.1016/j.cub.2005.01.022

Toth, K., Dawson, G., Meltzoff, A. N., Greenson, J., \& Fein, D. (2007). Early social, imitation, play, and language abilities of young non-autistic siblings of children with autism. Journal of Autism and Developmental Disorders, 37(1), 145-157. http://dx.doi.org/10.1007/s10803-006-0336-2

Van Gog, T., Paas, F., Marcus, N., Ayres, P., \& Sweller, J. (2009). The mirror neuron system and observational learning: Implications for the effectiveness of dynamic visualizations. Educational Psychology Review, 2l(1), 21-30. http://dx.doi.org/10.1007/s10648-008-9094-3

Wallen, M., \& Bulkeley, K. (2006). Three sessions of adult imitation increased some appropriate social behaviours of young children with autism. Australian Occupational Therapy Journal, 53(2), 139-140. http://dx.doi.org/10.1111/j.1440-1630.2006.00563.x

Williams, J. H., Whiten, A., Suddendorf, T., \& Perrett, D. I. (2001). Imitation, mirror neurons and autism. Neuroscience \& Biobehavioral Reviews, 25(4), 287-295. http://dx.doi.org/10.1016/S0149-7634(01)00014-8

Wilson, M., \& Knoblich, G. (2005). The case for motor involvement in perceiving conspecifics. Psychological Bulletin, 131(3), 460. http://dx.doi.org/10.1037/0033-2909.131.3.460

\section{Copyrights}

Copyright for this article is retained by the author(s), with first publication rights granted to the journal.

This is an open-access article distributed under the terms and conditions of the Creative Commons Attribution license (http://creativecommons.org/licenses/by/4.0/). 